\title{
The Dynamic Evolution Study of Technology Symbiosis
}

\author{
Fang $\mathrm{Li}^{1,2, a}$, Jianqi Mao ${ }^{1, b}$ \\ ${ }^{1}$ Shandong Normal University, Jinan, 250014, China \\ 2 Shandong Institute of Business and Technology, Yantai, 264005, China \\ alifang2013lf@163.com, ${ }^{\mathrm{b}}$ maojianqi2003@263.net
}

\begin{abstract}
The focus of technology research and development is to create value, the symbiosis theory provides a new perspective for studying technology innovation. Based on the symbiosis theory, this paper researches the occurrence conditions and the dynamic conditions. Then on the basis of technological symbiosis time-varying process, establish the dynamic evolution study of technology symbiosis. At last, this paper analyzes the change of technical symbiosis patterns according to the dynamic evolution process of technology symbiosis.
\end{abstract}

Keywords: technology symbiosis; technological stability; dynamic evolution.

\section{Introduction}

Parasitism, predation, symbiosis and competition are the prevalence of relationships among biologicals. The middle of the 19th century, it puts forward the concept of symbiosis on biology, however, the word "symbiosis" comes from the Greek ${ }^{[1]}$. Since then, the application fields of symbiosis theory are continuously extended, and symbiosis theory research problems are also getting deeper.

Technology plays a significant role in human society development, and is an important means to solve the problem of their own development. Just like one single creature depend on natural ecological system, there are close relationships between technology and technology, technology and environment-one single technology can not live without technology ecosystem. In the process of innovation and technology evolution, it is very necessary to research technology combining with related technology and environment factors ${ }^{[2]}$. The "Smiling Curve" tells us, Simple manufacture just a bottom part of the industrial chain, only innovation can create real benefits. How to make the research and development of product technology as well as management effectively, is an urgent problem to be resolved which influences China prompt from "made in China" to "created in China". Based on technology symbiosis theory, this paper from the micro perspective to explore the inherent law of technology development, hoping to provide the reference for enterprise technology research and development.

\section{Technology symbiosis conditions}

In biology, Symbiosis refers to different kinds of organisms in a certain contact live together in a certain environment. To promote to the general sense, symbiosis means a relationship that symbiotic unit in a certain symbiotic mode communicate material, information and energy with each other ${ }^{[3]}$.

Symbiosis contains symbiotic unit, symbiotic mode and symbiotic environment, namely three elements of symbiosis. The symbiosis between the biology is the result that species constantly adapt to complex natural environment and interact with other species in the process of long-term evolution. So how does the symbiotic relationship happen between technology and technology? Assumes that there are two symbiotic technologies $-\mathrm{A}$ and $\mathrm{B}$, and their qualitative parameters respectively are $Z_{a}$ and $Z_{b}$. The general conditions of technology symbiosis are:

\subsection{Qualitative parameters compatible with each other between symbiotic technologies}

The character and it's variation in symbiotic unit depend on qualitative parameters. Qualitative parameter compatible means qualitative parameter $Z_{a}$ and $Z_{b}$ could express to each other, making 
use of mathematical way: $Z_{a}=f\left(Z_{b}\right)$ or $Z_{b}=f\left(Z_{a}\right)$. That is to say, symbiotic units communicate information, material and energy with each other. For instance, the overall technology of smart phone and application processor technology can express each other, that is to say, there is a certain connection between the overall technology of smart phone and application processor technology.

\subsection{It generates symbiotic interface between symbiotic technologies.}

If the symbiotic technology exchange information, material and energy, it has to through symbiotic interface. Developing symbiotic technology system, increasing the number of symbiotic units and improving their quality, all of them are based on symbiotic energy production. Assuming that the total energy of symbionts is $\mathrm{E}$, and there are two symbiotic units A and $\mathrm{B}$. Under the condition of non symbiosis, the energies of $\mathrm{A}$ and $\mathrm{B}$ respectively are $Z_{a}$ and $Z_{b}$; under the condition of symbiosis, the new generated energy is $E_{s}{ }^{[4]}$. Then, the total energy is

$$
E=E_{a}+E_{b}+E_{s}
$$

The development of symbiosis system tells us, symbionts cannot proliferate and develop without symbiotic energy. Only generating symbiotic energy can it guarantee symbiosis system exist and progress.

\subsection{It has critical mass between symbiotic technologies.}

There is a certain critical value between symbiotic units. When the certain parameter of symbiotic unit no more than critical value, technology symbiotic relationship and symbiotic strength will be increased with the increase of the parameter, and even they reach to a peak; when the parameter greater than critical value, technology symbiotic relationship and symbiotic strength will be weakened with the increase of the parameter, and even lead to disintegration of symbionts.

\section{Technology symbiosis dynamic conditions}

In technology symbionts, the number of symbiotic technology is not always stable and it changes with the development of symbiont. In turn, the symbiont will be influenced by the number and kind of symbiotic technologies. In technology symbiosis, the change of a small parameter will lead to the variation of others ${ }^{[5]}$. In general, any kind of technology can't unlimited increase in symbiotic system. Before some point, the number of technology in the symbiotic system would increase constantly; and once exceeding the point, it would decrease. If the symbiont can achieve equilibrium and stability, how many symbiotic technologies that it must reach to?

\subsection{Symbiosis equilibrium conditions}

On the process of technology symbiosis, symbiotic technology generates symbiotic energy $E_{r}$ through the effect of symbiotic interface, at the same time, exists energy consumption $E_{c}$. Technology symbiotic dimension $\sigma$ reflects the number of heterogeneous symbiotic technologies; symbiotic density means the number of similar symbiotic units ${ }^{[6]}$. In that way, using mathematical method to solve marginal dimension of symbiosis energy and marginal dimension of symbiotic consumption is: $D_{\sigma}=\frac{\partial E_{r}}{\partial \sigma_{r}}, D_{\sigma c}=\frac{\partial E_{c}}{\partial \sigma_{c}}$.So, the condition of symbiotic dimension equilibrium is:

$$
D_{\sigma}=D_{\sigma}
$$

At this moment, $\frac{\partial E_{r}}{\partial \sigma_{r}}=0$, and $\Delta E_{r}$ exists the maximum. In the similar way, we can get the condition of symbiotic density equilibrium:

$$
D_{\rho c}=D_{\rho r}
$$


At this moment, $\frac{\partial E_{r}}{\partial \rho_{r}}=0$, and $\Delta E_{r}$ exists the maximum.

On the assumption that symbiotic density(dimension)reach to balance at $\rho_{e}\left(\sigma_{e}\right)$. We realize that when symbiotic density(dimension)is greater than $\rho_{e}\left(\sigma_{e}\right)$, the symbiotic energy decreases instead. That is to say, under the condition of the given resources and markets, the number of product technology cannot excessive inflation.

\subsection{Symbiosis stability conditions}

The stability of the symbionts cannot only measured by symbiotic unit of the total balance, however, the internal structure of symbionts characteristics determine whether the symbiosis stable ${ }^{[6]}$. As previously mentioned, the general condition of technology symbiosis is qualitative parameters compatible with each other. Namely, there is inevitable inner link between technologies. And the connection degree is higher, the symbiosis will be more stable.

On the process of symbiosis, the stability and development of symbiosis relationship depend on the allocation of symbiotic energy and consumption. The allocation condition to realize symbiosis stability is:

$$
\frac{E_{r a}}{E_{c a}}=\frac{E_{r b}}{E_{c b}}=k
$$

$E_{r}=E_{r a}+E_{r b}, E_{c}=E_{c a}+E_{c b} . \mathrm{k}$ is the distribution coefficient of symbiosis stability and a constant. At this time, the symbiont is in stable state of the best, and symbiotic energy distribution is symmetrical. The symbiosis mode is symmetry mutualism model, and this is the ideal state of symbiosis. When the distribution coefficient deviates from $\mathrm{k}$,

$$
k_{i}=k+\theta
$$

$\theta$ is a variable and deviation coefficient. This distribution is symmetrical, and symbionts present unstable state. When the distribution coefficient deviates to a certain degree even breaks through the critical value, symbionts is facing collapse.

Technology symbiotic dynamic evolution process analysis

Technology symbiosis is a dynamic and continuous improved process, which adapts to complicated environment and mutually accommodates other technologies. From the perspective of the symbiosis theory, Mao Jianqi put forward that "Technology ecology is symbiosis system"; analyzes technology symbiont, technology symbiotic conditions and time-varying process; proposes that "technology symbiosis experiences the symbiotic relationship of recognition, adaptation, development and disintegration of symbiosis as well as the formation of new symbiotic relationship; it is a dynamic process. "[7] With reference to his theory, this paper studies the dynamic evolution of technology symbiosis from the cyclic process--technology symbiosis "recognition, adaption, development, stabilization, disintegration of symbiosis as well as the formation of new symbiotic relationship".

According to the principle of quality parameter compatible, technology symbiosis must be a certain relationship between technologies. So, if there is identification between technology and technology, only have some connection or purpose could produce a symbiotic relationship between technologies. It is the early stages of technical symbiosis. Symbiotic relationship and the symbiotic interface extremely unstable, symbiotic energy and density are small; and $E_{r}>E_{c}$. Then enters the symbiotic adaptive phase. At this phase, technologies are unceasing developmental, and constantly adjust themselves to accommodate the symbiotic relationship; the stability of symbiotic relationship and the interface enhance, and coexistence densities within the symbionts begin to increase; symbiotic energy also start to increase, but the increase in overall energy is greater than consumption, namely $E_{r}>E_{c}$. After symbiosis adaption, Symbionts enter into the development stage of goldsymbiotic relationship development stage. At this stage, the stability of symbiotic interface and 
relationship strengthen rapidly, and coexistence densities increase sharply; the increment and consumption of energy also augment heavily, but $E_{r}>E_{c}$. Then it enters into the symbiotic relationship stable stage, when symbiosis develops to a certain degree; at this time, the stability of symbiotic interface and relationship reach to the maximum as well as density. In addition, marginal symbiosis density achieve equilibrium $D_{\rho c}=D_{\rho r}$. Energy distribution shows $\frac{E_{r a}}{E_{c a}}=\frac{E_{r b}}{E_{c b}}=k$-this is the best stable state. However, because of the different technical maturity and their own development, technology development or fast or slow, the stability of technology association will be broken; at this moment, the stability of symbiotic interface and relationship weakens constantly and the symbionts face to disintegration. Since then, technology individuals begin the new recognition according to a certain contact and a new round of symbiotic process. As shown in figure 1:

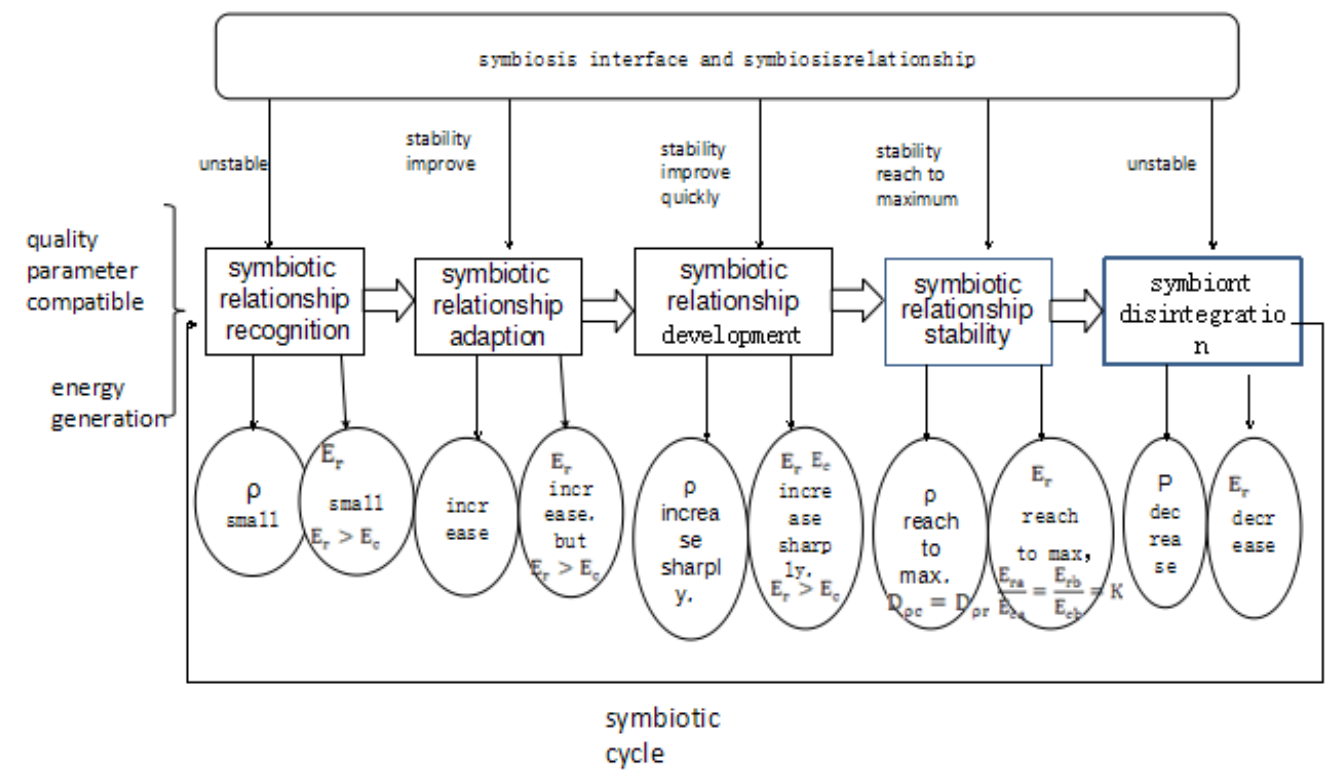

Figure 1 Technology symbiotic dynamic evolution model

\section{The behavior patterns study in symbiosis dynamic evolution}

Yuan Chunqing ${ }^{[6]}$ divides symbiotic modes into four behavior patterns___parasitic, partial symbiosis, asymmetry mutualism and symmetric symbiosis ". As shown in table 1:

Table 1 technology symbiosis modes

\begin{tabular}{|c|c|}
\hline Symbiosis modes & Symbiont characters \\
\hline parasitic & generate energy, parasitic body asks for energy from the host uniaxially \\
\hline partial symbiosis & $\begin{array}{c}\text { generate energy ; bidirectional communication; only one side gets whole } \\
\text { energy }\end{array}$ \\
\hline $\begin{array}{l}\text { asymmetry } \\
\text { mutualism symbiosis }\end{array}$ & $\begin{array}{l}\text { Generate energy; multilateral and multidirectional communication; but the } \\
\text { energy distribution is asymmetric and evolution is not synchronous. }\end{array}$ \\
\hline symmetric symbiosis & $\begin{array}{l}\text { Generate energy; energy distribution is symmetric, multilateral } \\
\text { communication; symbiotic technologies evolve synchronous. }\end{array}$ \\
\hline
\end{tabular}

Symbiosis is a process that based on symbiotic interface, symbiotic units exchange material, information and energy and generate symbiotic effect. According to the principle of quality parameter compatible, the more closely technology symbiotic units contact, the less marginal density of symbiosis consume and the more stable the symbiont is. In addition, symbiotic energy allocation determines the symbiotic stability. In the early stages of producing technology to produce symbiosis, due to the weak of assistant or support technology, it can only obtain energy from the core one in order to maintain its development. At this time, the symbiotic mode is a parasite. With the development of technology, factors develop increasingly perfect such as symbiotic interface and 
symbiotic environment. When the ratio of symbiotic energy and energy loss is equal in technology symbiotic unit, that is $\frac{E_{r a}}{E_{c a}}=\frac{E_{r b}}{E_{c b}}=k$, Symbiont is in stable state, which the energy distribution is symmetrical and symbiotic mode presents asymmetry mutualism. However, when the ratio is inequal and deviates, symbiosis energy distribution is asymmetric even partial. So at that moment, symbiotic mode becomes the asymmetric mutualism and even partial symbiotic model, and the symbiotic mode of symbionts is unstable. At this point, symbiotic unit would judge factors such as symbiotic interface, internal structure and energy distribution, whether can further symbiosis. If so, then the symbiotic units adjust the symbiotic mode by improving the efficiency of the symbiotic interface, optimizing the allocation of energy etc, and promote the symbiotic mode to a more ideal one evolve. Otherwise, symbionts will collapse.

\section{Conclusion}

Technology symbiosis is the intrinsic and inevitable characteristic of technology. The development of symbionts is not set in stone. In the process of symbiosis identification, adaptation, development, collapse and new symbiotic relationship formation, the density of symbionts and energy distribution are changing; the symbiotic interface and symbiotic relationship are from stable to unstable then to stable. However, it is for these changes that the development of the symbionts could be promoted. From the micro perspective, this paper reveals changes of symbionts in the process of technology dynamic evolution; tries to uncover the "black box" in the process of technological innovation and provides new ideas for enterprise R\&D.

\section{Acknowledgment}

This paper based on the national natural science foundation (71172086).Li fang, born in 1990, female, shandong normal university, master, research direction of technology and innovation management. Many thanks to Mao Jian (born in 1963, male, shandong business school professor, doctor, master tutor, the research direction of technology and innovation management).

\section{References}

[1]Yang Bowen, Huang Hengzhen. Symbiosis Theory: the New Foundation of Organization Evolution [J]. Journal of university of electronic science and technology (social science edition), 2010, 12(2):29-32.

[2] Gediminas Adomavicius,Jesse C. Bockstedt,,Alok Gupta, Robert J. Kauffman. Making sense of technology trends in the information technology landscape: a design science approach [J]. MIS Quarterly - Management Information Systems 2008, 32(4):779-810.

[3]Wang Yulu. Bank symbiosis mechanism and symbiotic pattern and symbiosis theory research [J].Shanghai finance, 2007,1: 25-28.

[4]Si Shangqi, Cao Zhenquan, Feng Feng.: Research institutions and enterprise symbiosis mechanism research-based on the symbiosis theory and frame [J].Science, and science and technology management, 2009, 6:15-19.

[5]Baldwin, C., Clark, K., 2000. Desigs: the power of modularity. MIT Press,, Cambridge, MA.

[6]Yuan Chunqing. Symbiosis theory_—and the theory of small economy [M].Beijing: Economic science press, 1998:5-18.

[7]Mao Jianqi, Liu Na, Chen Lei. Mechanism study on technology symbiosis —an explanatory framework based on symbiosis theory [J]. Dialectics of nature, 2011, 27(6):36-41. 